\title{
Epithelial Brush Border Proteomics and Associated Cell Dysfunction
}

\author{
Xiangdong Wang*,1 and Kenneth B. Adler ${ }^{2}$ \\ ${ }^{I}$ Department of Respiratory Medicine, Zhongshan Hospital, Fudan University, Shanghai, China \\ ${ }^{2}$ Department of Molecular Biomedical Sciences, North Carolina State University, Raleigh, NC, USA
}

\begin{abstract}
Functions and structures of epithelial cells vary, depending upon their locations in organs/tissues. Pathophysiological responses of epithelia are associated with challenges, severities, durations and diseases. Epithelial proteomics has been used to understand the role of these cells in the pathogenesis of several diseases, and has the potential to identify novel biomarkers for disease prognosis, diagnosis and therapies. Epithelial brush border membranes (BBMs) are involved in digestion, absorption, metabolism, and transport of nutrients and drugs, clearance of and defense against toxins, and initiation of intracellular signaling and maintenance of cellular structural integrity. The present article review proteomic research on epithelial brush borders to understand the potential links among the function and disease associations of epithelial BBM proteomics. We found that the amount and functional ratio of epithelial cell BBM proteomic profiles obviously vary among species, cell locations, organ functions, research groups, and methodologies utilized. Many of these proteins may be identified and their functions elucidated, probably providing potential new markers for epithelial-associated diseases and potential therapeutic targets. There is still a great need for proteomic studies for analysis and comparisons of BBM protein profiles associated with normal physiological and pathophysiological states, organ function and dysfunction, and clinical health and disease.
\end{abstract}

Keywords: Epithelium, proteome, microvilli, brush border membrane.

\section{INTRODUCTION}

Polarized epithelial cells in multiple organs confront the environment with a highly and structurally specialized apical cell membrane, referred to microvilli or brush border membranes (BBMs), which differ in composition and function from epithelial surfaces that face the internal milieu. Many cells throughout the body have developed "microvillous" appendages for various tasks, including sensing fluid flow (through renal distal tubules), absorption, chemosensing, or repair of ciliated cells after injury. Brush cells, also termed tuft, caveolated, multivesicular, and fibrillovesicular cells, are defined as part of the epithelial layer in the gastrointestinal and respiratory tracts [1]. The focus of this article is on a broad (airway, gastrointestinal, renal, retinal and others) of epithelial cells with microvilli and BBM. BBMs are responsible for digestion, absorption, metabolism, and transport of nutrients and drugs, for clearance of and defense against toxins, and for initiation of intracellular signaling and maintenance of cellular structural integrity. BBMs vary between organs and between cells in the same organ/tissue. For example, airway ciliated and non-ciliated columnar cells are covered with microvilli, with fine electron-dense filaments identifiable in the cytoplasm running parallel to the long axis [2]. Microvilli on non-ciliated cells are thicker than those of the ciliated cells.

Epithelial protein profiles have been associated with intracellular locations and protein functions. Increased numbers of airway brush cells have been described in a human

*Address correspondence to this author at the Department of Respiratory Medicine, Zhongshan Hospital, Fudan University, Shanghai, China;

E-mail: Xiangdong.wang@telia.com infant with desquamative interstitial pneumonitis and in airways of individuals with immotile cilia syndrome $[3,4]$.

Epithelial proteomics has been found to have a strong link with clinical questions, e.g., disease severity, biomarkers for disease diagnosis, and drug targets [5]. Epithelial proteomics has the potential to make a major contribution to our understanding of the pathogenesis of organ dysfunction when combined with clinical measures, tissue imaging and profiling, and organ dysfunction score systems [6]. Epithelial proteomic profiles have been investigated in multiple pathophysiological conditions, including cancer, inflammation, stress, infection, and others [7-10]. BBMs are considered the first line of defense of epithelial cells against inhaled external elements, and it is known that interaction with such stimuli can trigger intracellular signals. It would be useful to have a number of biology-, pharmacology- and diseasespecific markers for the BBM for better understanding of cellular function and metabolism, drug efficacy and toxicity validation, and pathogenesis of and new therapies for diseases. The present article reviews protein profiles and functional ratios of BBM, compares the similarities and differences of BBM proteomics among cells, discusses the potential biological significance of some identified BBM proteins, and explores the association with and involvement of BBMs in the pathogenesis of disease.

\section{PROTEIN FUNCTIONAL RATIO}

Protein profiles of human BBMs and their physiological and pathophysiological significances are still unclear. The native membrane proteome of BBM was analyzed in separated protein complexes from purified mouse intestinal BBMs by the blue native PAGE (BN-PAGE) technique [11]. About 55 proteins were identified from 23 distinct protein 
complexes in intestinal epithelial BBMs, including membrane transporters $(21 \%)$, signal transduction $(16 \%)$ and cytoskeletal $(15 \%)$ proteins. Protein profiles of syncytiotrophoblast BBMs in the placenta of human embryos mainly include signal transduction (34\%), cell growth and/or maintenance $(30 \%)$, and membrane transporters (16\%) [12]. About 45 surface proteins were identified in human mammary epithelial cells (strain 184 A1L5) using lysine specific in situ labeling of the proteins via sulfosuccinimidyl-6- (biotinamido) hexanoate [13]. According to protein transmembrane and signal sequence properties, $9 \%$ of the proteins contained both transmembrane domains and signal sequences, $56 \%$ of the proteins only a transmembrane domain, $3 \%$ of the proteins only a signal sequence, and $33 \%$ of the proteins at the surface of the epithelium of the mammalian oviduct were "others" [14]. The majority of proteins of the BBMs of rat renal proximal tubule epithelial cells were found to be related to metabolism (20\%) and signal transduction (10\%) [15]. Proteomic profiles of mouse jejunal BBM showed a relation to signal transcription $(25 \%)$, metabolism $(21 \%)$, cytoskeleton $(16 \%)$ and membrane transport $(11 \%)$ [7].

The comparison of the ratio of protein profiles associated with function (Table 1) between these cells indicates that BBMs contribute to cell signaling and absorption/secretion/metabolism, In intestinal cells, BBMs are more involved in structural functions, while proteins associated with cell/tissue development and lipid rafts characterize BBMs of syncytiotrophoblast, and BBMs of renal cells contain mostly proteins associated with metabolism. The amount and functional ratio of epithelial cell BBM proteomic profiles obviously vary among species, cell locations, organ functions, research groups, and methodologies used. For example, the number of identified proteins separated from sodium dodecyl sulfate-polyacrylamide gel electrophoresis is almost double that from cation-exchange high-performance liquid chromatography [15]. The number of proteins identi-

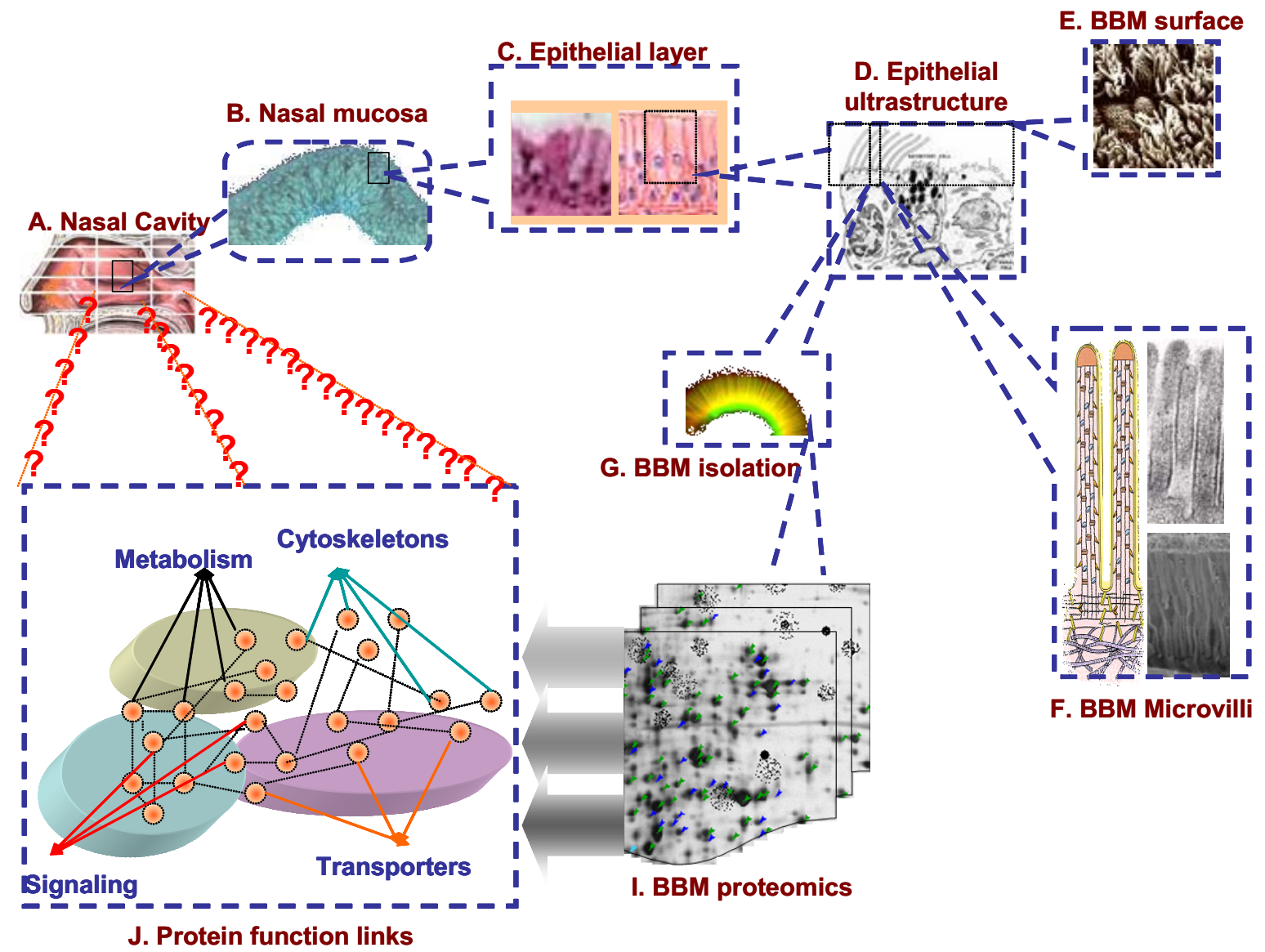

Fig. (1). The influencing process of epithelial brush border membrane (BBM) proteomic analysis. A: The mucosal tissue is harvested from the body (e.g. nasal mucosa); epithelial structure and function vary among locations and organs. B and C: mucosal histology provides evidence of mucosal (B) and epithelial (C) similarity and variation prior to the isolation of epithelial BBMs, in order to ensure that the result are comparable. D-F: The epithelial ultrastructure from both (F) transmission (D) and scanning (E) electronic microscopy demonstrates variations between epithelial cells from the same location. Epithelial cells have three domains: apical, lateral and basal. Of these, the apical domain $(B B M)$ is a free surface always directed toward the exterior or lumen of the enclosed body cavity, having surface modifications depending upon specific function. G: Epithelial BBM is isolated from the cells, probably including microvilli, stereocilia and cilia. It should be noted that potential variation exists between BBMs, even though they appear similar microscopically. I: BBM proteomic analysis can be performed by different methodologies, which describe the different amounts and sizes of protein profiles. $\mathbf{J}$ : one of the important steps is to link these identified proteins with biological functions. Although a number of studies on epithelial BBM proteomics have shown similarities and variations in these functional distributions between epithelia, there is a great need to translate such information to clinical sciences and understand more about how this information relates to disease pathogenesis. 
Table 1. Functional Distribution of the Proteins Identified in Brush Border Membrane Preparations of Intestinal, Renal, Retinal, Jeunal and Syncytiotrophoblast Epithelia

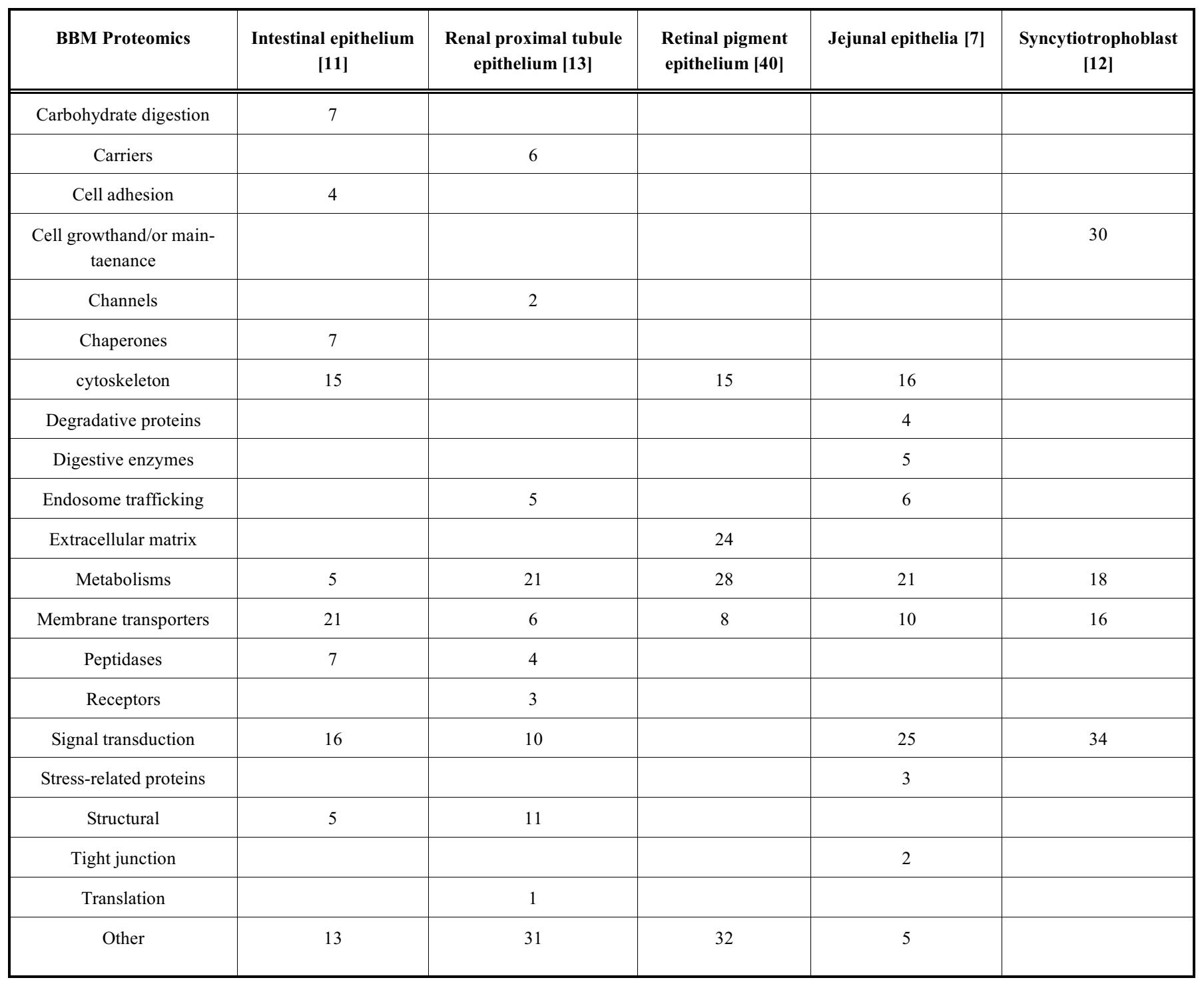

fied using the multidimensional protein identification technology (MudPIT) is approximately 7 times higher than that identified by 2D gel electophoresis in the same samples [14]. Table 2 lists these identified protein profiles from epithelial BBMs, showing variations and similarities of proteomic profiles between different epithelia.

The percentage of BBM-related proteins in epithelial cells varies between about $10-25 \%$ of the total membraneassociated proteins $[16,17]$ depending upon the technology used for preparation and separation of BBM proteins, the epithelial origin, modifications of cellular genes, and treatment used on the cells. For example, it was found that about $15 \%$ of the cell surface proteins could be biotinylated with sulfo-NHS-S-S-biotin, $8 \%$ could be glycosylated with lectinaffinity, and $77 \%$ made up the remaining protein populations in human mammary epithelial cell BBMs transfected with a recombinant EGF gene [17]. It would be more important to standardize the methodology of BBM isolation, protein separation, and proteomic analysis in the future in order to make valid comparisons of the proteomics of epithelial BBMs, which can make understanding of the role(s) of BBMs in disease pathogenesis even more significant.

\section{BIOLOGICAL SIGNIFICANCE}

BBM protein profiles can vary with different challenges and environments. For example, syndecan-4, a transmembrane heparin sulfate proteoglycan, and hepatoma-derived growth factor (HDGF), a nuclear protein, are increased in BBMs when epithelial cells are exposed to the phorbol ester, 4b-phorbol 12-myristate 13-acetate [17]. This indicates that epithelial BBMs shed and become active in signal transduction, since syndecan- 4 is involved in both constitutive and regulated mechanisms [18] and HDGF as an extracellular heparin-binding growth factor can bind to a membrane protein(s) stimulating growth-regulatory signal transduction [19]. It was suggested that HDGF could act as a bioactive cell surface protein that may be actively processed by regulated proteolysis.

HDGF is a nuclear targeted protein containing a canonical bipartite nuclear localization sequence. It has been con- 
Table 2. Proteomic Profiles of Intestinal, Syncytiotrophoblast, Retinal Pigment, Marramary Epithelail Brush Border Membrane

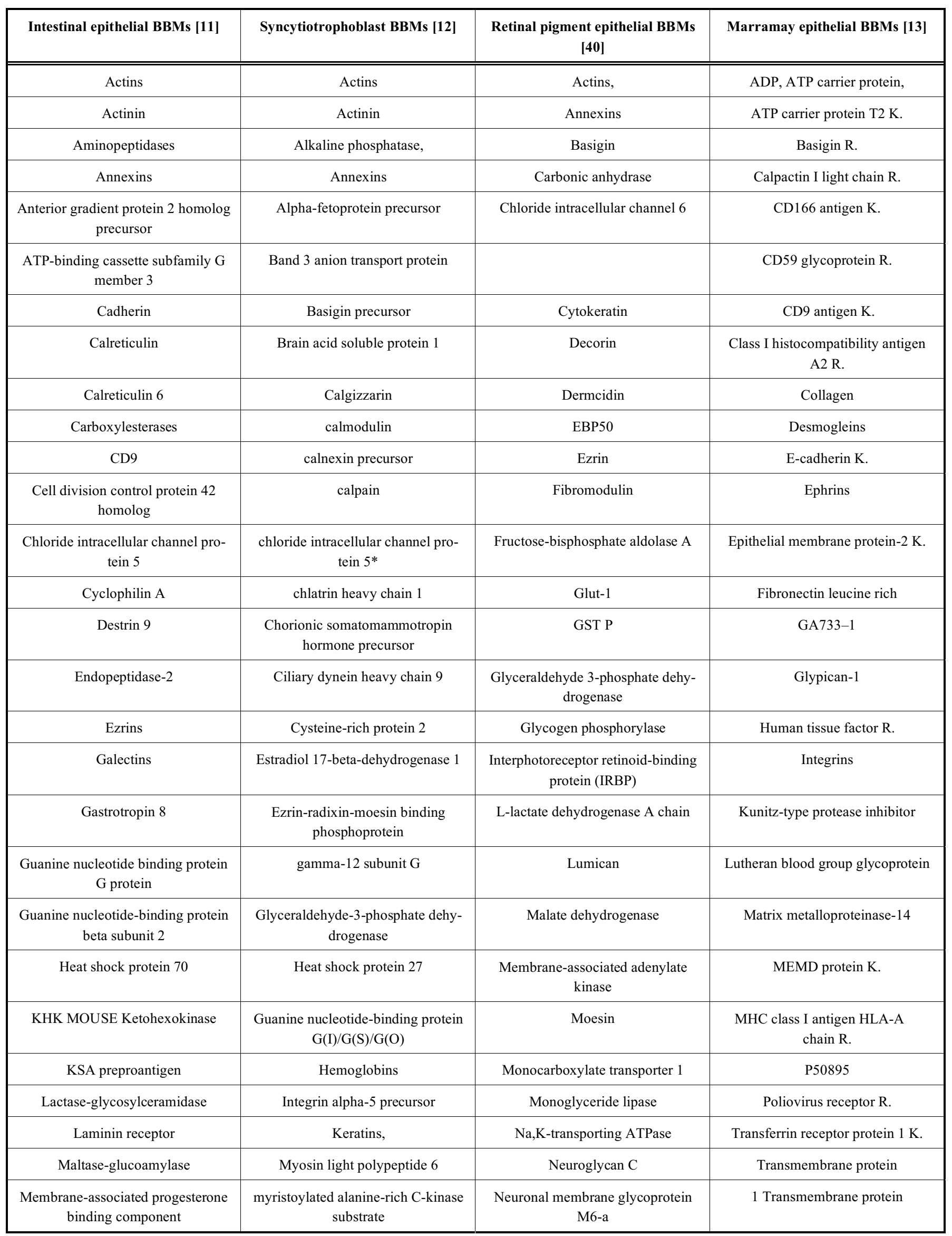


(Table 2). Contd.....

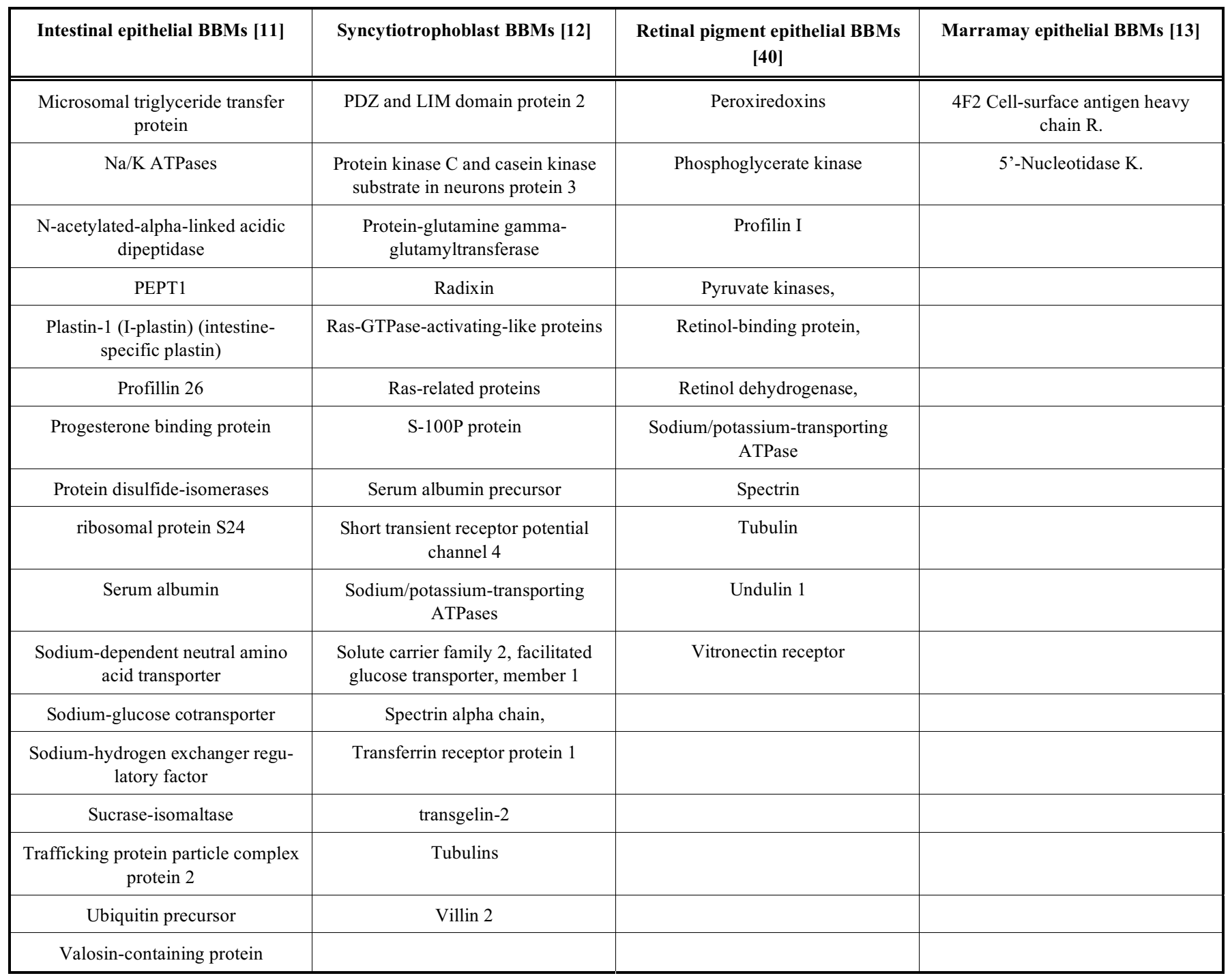

sidered a novel biomarker or prognostic factor in multiple diseases, such as gastrointestinal stromal tumors [20], pancreatic cancer [21], vascular injury [22], and hepatocellular carcinoma [23], and is correlated with tumor recurrence in esophageal carcinoma [24]. These experimental and clinical studies demonstrated that expression of HDGF is significantly increased in disease and correlates with the incidence and recurrence of cancers These studies did not clarify the source of HDGF, which normally is found in low concentrations in multiple cells (e.g. epithelial cells, endothelial cells and smooth muscle cells). Although it is difficult to envision that HDGF is released from epithelial BBMs, most cancers originate from epithelial cells, and thus HDGF has been suggested as a potential target for anti-cancer drug design and development [25].

Lipid rafts are a type of heterogeneous functional microdomain of the plasma membrane enriched in glycosphingolipids/cholesterol and specific proteins [26]. Lipid rafts are believed to play a role in major mechanisms of membrane trafficking, transport of glycosyl phosphatidylinositol-anchored proteins and glycosphingolipids to the cell surface, nutrient absorption, regulated secretion, and transport from endosomes to the Golgi apparatus and internalization via both caveolae and clathrin-coated pits. Proteomic analyses have been used to characterize protein profiles of epithelial BBMs for lipid raft markers [27,28], and characterization of lipid raft markers showed the presence of $\mathrm{N}$-aminopeptidase, alkaline phosphatase, dipeptidyl aminopeptidase, annexin II, galectin-4, GP2, annexin IV, XIIIb, Gaq, Ga11, glutamate receptor, and GPCR 7 [27], indicating that some digestive enzymes, trafficking and signaling proteins may be functionally distributed in the intestine lipid rafts. Lipid rafts have been considered to associated with a number of diseases, e.g. severe congenital myopathy, colitis, cholera, autoimmune diseases (systemic lupus erythematosus and rheumatoid arthritis), virus infection, cancer, insulin resistance, inflammation, cardiovascular disease, hypertension, systemic lupus erythematosus, and others [29-33]. However, it remains unclear how these lipid raft markers might link directly with the pathogenesis of disease, whether they can be diseasespecific biomarkers to predict and monitor the development, severity and prognosis of the disease, or what role they may play in the molecular mechanisms of the disease process. It is also important to clarify whether these lipid raft markers are truly lipid raft-specific proteins. 
When compared proteomic profiles between plasma membranes from proliferating cells and apical membranes from differentiated cells, 76 proteins were found to be significantly increased in the membranes of differentiated cells and 61 were increased in proliferating cells [34]. The majority of the proteins increased in the apical membranes were metabolic enzymes, proteins involved in the maintenance of cellular structure, transmembrane transporters, and proteins regulating vesicular transport, while the majority in the plasma membrane were involved in gene expression, protein synthesis, and folding [34]. It was suggested that many of these proteins may have specific functions and may provide potential new markers of intestinal cells or of colorectal cancer. The potential links between significantly increased proteins from differentiated cells, protein biology, and associated consequences are listed in Table $\mathbf{3}$.

\section{SIGNIFICANCE IN DISEASES}

Functional identification and disease-associated evaluation of protein profiles found from proteomic studies are varied. There is still a great need for proteomic studies for analysis and comparisons of BBM protein profiles between cell physiological and pathophysiological situations, organ function and dysfunction, and clinical health and disease. Although there is much current research describing proteomic profiles of BBM harvested from various epithelia, it is possible to explore a potential link with biology. For example, Table 3 lists a number of identified and significantly changed proteins identified from BBMs of differentiated cells as compared with those from proliferating cells [35], some of which are involved in the pathogenesis of disease, while the function of others are still unclear. These changed proteins become even more important and significant when

Table 3. The Potential Links Between Significantly Increased Proteins from Differentiated Cells, Protein Biology, and Associated Consequences; Examples are from Identified Proteins in a Published Study [35]

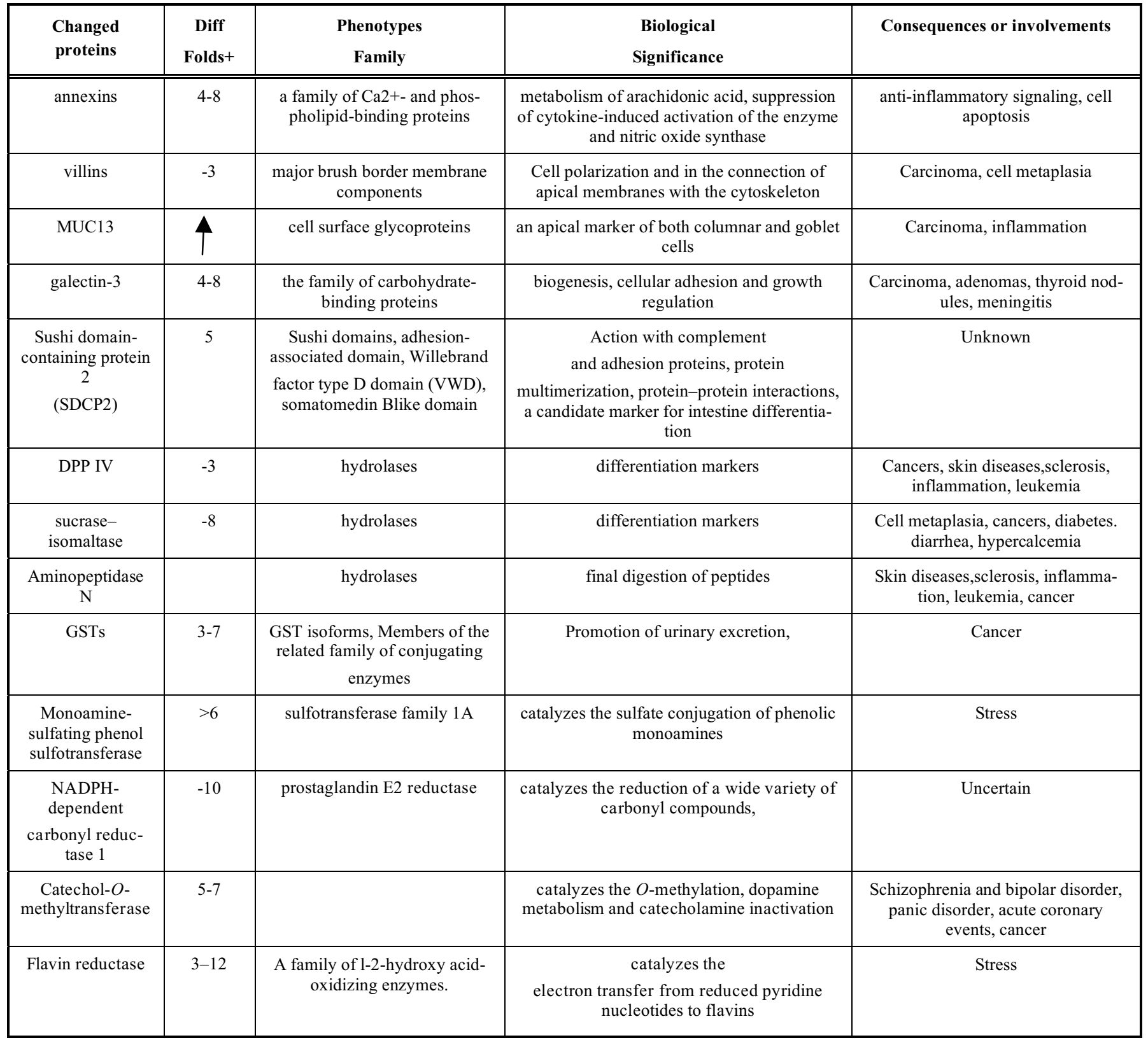


protein-associated pathophysiology is explored. Annexin 1 has been suggested to have anti-inflammatory effects, and may act by influencing the metabolism of arachidonic acid, targeting cytosolic PLA2, inhibiting nitric oxide synthase and neutrophil and monocyte migration, and/or promoting inflammatory cell apoptosis [36]. Galectin-3, a family of carbohydrate-binding proteins with high affinity for galactoside, is involved in cell growth and differentiation, cell adhesion, tumor progression, apoptosis and metastasis. Galectin-3 was found to be highly expressed in functioning corticotroph adenomas of the pituitary gland, and is used in pathological diagnosis to separate functioning from silent corticotroph adenomas of the pituitary [37]. Dipeptidyl peptidase-IV (DPP-IV) has a unique proteolytic activity, cleaving $\mathrm{N}$ terminal X-Pro dipeptides which may be involved in formation of cancers (e.g. the expression and possible function of DPP-IV enzymatic activity bearing molecules in human brain tumors has been addressed) [38]. Inhibitors of DPP-IV and aminopeptidase $\mathrm{N}$ were found to affect proliferation, differentiation and cytokine production in epithelia, to suppress $\mathrm{T}$ cell-stimulated proliferation, and to induce an antiinflammatory cytokine profile, while inhibition of DP IVlike activity only reduced the TGF-beta1 mediated stimulatory effects [39]. This indicates that similarities and variations between these proteins should be paid special attention if selecting a novel approach for treatments or therapies. For example, deficiency of sucrase isomaltase in patients could result in the development of intestinal metaplasia, cancers, diabetes, diarrhea, hypercalcemia and, in infants, chronic protracted diarrhea [34].

In conclusion, the amount and functional ratio of epithelial cell BBM proteomic profiles vary among species, cell location and type, organ function, investigators and methods used. It has been suggested that many of these proteins may have novel identifiable functions, and may provide potential new markers for epithelial-associated diseases. There is still a great need for proteomic studies to analyze and compare BBM protein profiles between cell physiological and pathophysiological situations, organ function and dysfunction, and clinical health and disease.

\section{ACKNOWLEDGEMENT}

This is sponsored by Shanghai Leading Academic Discipline Project, NO B115.

\section{REFERENCES}

[1] Reid L, Meyrick B, Antony VB, Chang LY, Crapo JD, Reynolds HY. The mysterious pulmonary brush cell: a cell in search of a function. Am J Respir Crit Care Med 2005; 172: 136-9.

[2] Davis AE, Smallman LA. An ultrastructural study of the mucosal surface of the human inferior concha. I. Normal appearances. J Anat 1988; 161: 61-71.

[3] DiMaio MF, Dische R, Gordon RE, Kattan M. Alveolar brush cells in an infant with desquamative interstitial pneumonitis. Pediatr Pulmonol 1988; 4: 185-91.

[4] Gordon RE, Kattan M. Absence of cilia and basal bodies with predominance of brush cells in the respiratory mucosa from a patient with immotile cilia syndrome. Ultrastruct Pathol 1984; 6: 4559.

[5] Zhao H, Adler KB, Bai C, Tang F, Wang XD. Epithelial proteomics in multiple organs and tissues: similarities and variations between cells, organs, and diseases. J Proteome Res 2006; 5: 74355.
[6] Wang XD, Adler KB, Chaudry IH, Ward PA. Better understanding of organ dysfunction requires proteomic involvement. J Proteome Res 2006; 5: 1060-2.

[7] An HJ, Kim DS, Park YK, et al. Comparative proteomics of ovarian epithelial tumors. Proteome Res 2006; 5: 1082-90.

[8] Werner T, Shkoda A, Haller D. Intestinal epithelial cell proteome in IL-10 deficient mice and IL-10 receptor reconstituted epithelial cells: impact on chronic inflammation. J Proteome Res 2007; 6: 3691-3704.

[9] Yan Y, Weaver VM, Blair IA. Analysis of protein expression during oxidative stress in breast epithelial cells using a stable isotope labeled proteome internal standard. Proteome Res 2005; 4: 20072014.

[10] Das S, Sierra JC, Soman KV, et al. Differential protein expression profiles of gastric epithelial cells following Helicobacter pylori infection using ProteinChips. J. Proteome Res 2005; 4: 920-30.

[11] Babusiak M, Man P, Petrak J, Vyoral D. Native proteomic analysis of protein complexes in murine intestinal brush border membranes. Proteomics 2007; 7: 121-9.

[12] Paradela A, Bravo SB, Henríquez M, et al. Proteomic analysis of apical microvillous membranes of syncytiotrophoblast cells reveals a high degree of similarity with lipid rafts. J Proteome Res $2005 ; 4$ : 2435-41.

[13] Chen WN, Yu LR, Strittmatter EF, Thrall BD, Camp DG, Smith $\mathrm{RD}$. Detection of in situ labeled cell surface proteins by mass spectrometry: application to the membrane subproteome of human mammary epithelial cells. Proteomics 2003; 3: 1647-51.

[14] Sostaric E, Georgiou AS, Wong CH, Watson PF, Holt WV, Fazeli A. Global profiling of surface plasma membrane proteome of oviductal epithelial cells. J Proteome Res 2006; 5: 3029-37.

[15] Cutillas PR, Biber J, Marks J, et al. Proteomic analysis of plasma membrane vesicles isolated from the rat renal cortex. Proteomics 2005; 5: 101-12.

[16] Jacobs JM, Mottaz HM, Yu LR, et al. Multidimensional proteome analysis of human mammary epithelial cells. J Proteome Res 2004; 3: 68-75.

[17] Ahram M, Adkins JN, Auberry DL, Wunschel DS, Springer DL. A proteomic approach to characterize protein shedding. Proteomics 2005; $5:$ 123-31.

[18] Fitzgerald ML, Wang Z, Park PW, Murphy G, Bernfield M. Shedding of syndecan-1 and -4 ectodomains is regulated by multiple signaling pathways and mediated by a TIMP-3-sensitive metalloproteinase. J Cell Biol 2000; 148: 811-824.

[19] Enomoto H, Yoshida K, Kishima Y, et al. Hepatoma-derived growth factor is highly expressed in developing liver and promotes fetal hepatocyte proliferation. Hepatology 2002; 36: 1519-27.

[20] Chang KC, Tai MH, Lin JW, et al. Hepatoma-derived growth factor is a novel prognostic factor for gastrointestinal stromal tumors. Int J Cancer 2007; 121: 1059-65.

[21] Uyama $\mathrm{H}$, Tomita $\mathrm{Y}$, Nakamura $\mathrm{H}$, et al. Hepatoma-derived growth factor is a novel prognostic factor for patients with pancreatic cancer. Clin Cancer Res 2006; 12: 6043-8.

[22] Narron JV, Stoops TD, Barringhaus K, Matsumura M, Everett AD. Hepatoma-derived growth factor is expressed after vascular injury in the rat and stimulates smooth muscle cell migration. Pediatr Res 2006; 59: 778-83.

[23] Yoshida K, Tomita Y, Okuda Y, et al. Hepatoma-derived growth factor is a novel prognostic factor for hepatocellular carcinoma. Ann Surg Oncol 2006; 13: 159-67.

[24] Yamamoto S, Tomita Y, Hoshida Y, Morii E, Yasuda T, Doki Y. Expression level of hepatoma-derived growth factor correlates with tumor recurrence of esophageal carcinoma. Ann Surg Oncol 2007; 14: $2141-9$.

[25] Everett AD, Bushweller J. Hepatoma derived growth factor is a nuclear targeted mitogen. Curr Drug Targets 2003; 4: 367-71.

[26] Simons K, Toomre D. Lipid rafts and signal transduction. Nat Rev Mol Cell Biol 2000; 1: 31-39.

[27] Danielsen EM, Hansen GH. Lipid raft organization and function in brush borders of epithelial cells. Mol Membr Biol 2006; 23: 71-9.

[28] Danielsen EM, Hansen GH. Lipid rafts in epithelial brush borders: atypical membrane microdomains with specialized functions. Biochim Biophys Acta 2003; 1617: 1-9.

[29] Kajor M, Wojaczyńska-Stanek K, Marszał E, GrzybowskaChlebowczyk U, Fidziańska A. Lipid raft disease? A new severe congenital myopathy. Folia Neuropathol 2007; 45: 242-6. 
[30] Li Q, Zhang Q, Zhang M, et al. Effect of n-3 polyunsaturated fatty acids on membrane microdomain localization of tight junction proteins in experimental colitis. FEBS J 2008; 275: 411-20.

[31] Saslowsky DE, Lencer WI. Conversion of apical plasma membrane sphingomyelin to ceramide attenuates the intoxication of host cells by cholera toxin. Cell Microbiol 2008; 10: 67-80.

[32] Jury EC, Flores-Borja F, Kabouridis PS. Lipid rafts in T cell signalling and disease. Semin Cell Dev Biol 2007; 18: 608-15.

[33] Ma DW. Lipid mediators in membrane rafts are important determinants of human health and disease. Appl Physiol Nutr Metab 2007; 32: $341-50$.

[34] Reinshagen K, Keller KM, Haase B, Leeb T, Naim HY, Zimmer KP. Mosaic pattern of sucrase isomaltase deficiency in two brothers. Pediatr Res 2008; 63: 79-83.

[35] Pshezhetsky AV, Fedjaev M, Ashmarina L, et al. Subcellular proteomics of cell differentiation: quantitative analysis of the plasma membrane proteome of Caco-2 cells. Proteomics 2007; 7: 2201-15.
[36] Parente L, Solito E. Annexin 1: more than an anti-phospholipase protein. Inflamm Res 2004; 53: 125-32.

[37] Thodou E, Argyrakos T, Kontogeorgos G. Galectin-3 as a marker distinguishing functioning from silent corticotroph adenomas. Hormones 2007; 6: 227-32.

[38] Busek P, Stremenova J, Sedo A. Dipeptidyl peptidase-IV enzymatic activity bearing molecules in human brain tumors--good or evil? Front Biosci 2008; 13: 2319-26.

[39] Thielitz A, Ansorge S, Bank U, et al. The ectopeptidases dipeptidyl peptidase IV (DP IV) and aminopeptidase N (APN) and their related enzymes as possible targets in the treatment of skin diseases. Front Biosci 2008; 13: 2364-75.

[40] Bonilha VL, Bhattacharya SK, West KA, et al. Proteomic characterization of isolated retinal pigment epithelium microvilli. Mol Cell Proteomics 2004; 3: 1119-27.

(C) Wang and Adler; Licensee Bentham Open.

This is an open access article distributed under the terms of the Creative Commons Attribution License (http://creativecommons.org/licenses/by/2.5/), which permits unrestrictive use, distribution, and reproduction in any medium, provided the original work is properly cited. 